\title{
Social deprivation and prognosis in Scottish patients with pulmonary arterial hypertension
}

\author{
Katherine Pellino ${ }^{1}$, Simon Kerridge ${ }^{2}$, Colin Church ${ }^{2}$, Andrew J. Peacock ${ }^{2}$, \\ Timothy Crowe ${ }^{2}$, Geeshath Jayasekera ${ }^{2}$, Martin K. Johnson ${ }^{2,3}$ and \\ Alison M. MacKenzie ${ }^{2,3}$
}

\begin{abstract}
Affiliations: ${ }^{1}$ University of Wisconsin School of Medicine and Public Health, Madison, Wisconsin, USA. ${ }^{2}$ Scottish Pulmonary Vascular Unit, Golden Jubilee National Hospital, Glasgow, UK. ${ }^{3}$ Both authors contributed equally.
\end{abstract}

Correspondence: Martin K. Johnson, Scottish Pulmonary Vascular Unit, Golden Jubilee National Hospital, Agamemnon Street, Clydebank, G81 4DY, UK. E-mail: mjohnson4Qnhs.net

@ERSpublications

Social deprivation is not a significant referral barrier or prognostic factor for IPAH and HPAH in Scotland http://ow.ly/N7aL30gE4ui

Cite this article as: Pellino K, Kerridge S, Church C, et al. Social deprivation and prognosis in Scottish patients with pulmonary arterial hypertension. Eur Respir J 2018; 51: 1700444 [https://doi.org/10.1183/ 13993003.00444-2017].

ABSTRACT Several demographic and clinical factors have prognostic significance in idiopathic pulmonary arterial hypertension (IPAH). Studies in China and the USA have suggested an association between low socioeconomic status and reduced survival. The impact of social deprivation on IPAH survival in the UK is not known.

280 patients with IPAH and hereditary PAH (HPAH) attending the Scottish Pulmonary Vascular Unit (Glasgow, UK) were assigned to social deprivation quintiles using the Scottish Index of Multiple Deprivation database. The association between survival and social deprivation quintile was assessed using Cox proportional hazards regression analysis.

The distribution of IPAH/HPAH patients was more socially deprived than would be expected based on Scottish citizenry as a whole (Chi-squared 16.16, $\mathrm{p}=0.003$ ), suggesting referral and access to care is not impeded by socioeconomic status. Univariate analysis demonstrated no significant association between social deprivation and survival $(\mathrm{p}=0.81)$, and this association failed to reach significance with inclusion of time, sex and age as covariates in the model $(\mathrm{p}=0.23)$. There were no statistically significant correlations between social deprivation and baseline clinical variables of prognostic importance except for age, sex and quality of life.

Social deprivation is not a significant referral barrier or prognostic factor for IPAH and HPAH in Scotland.

This article has supplementary material available from erj.ersjournals.com

Received: March 012017 | Accepted after revision: Nov 052017

Conflict of interest: None declared.

Copyright @ERS 2018 


\section{Introduction}

Social deprivation is a measure of social wellbeing as it relates to factors such as employment, education and access to healthcare. As part of a holistic model of disease, social factors have been found to play an important role in determining the course of illness. Investigations into the impact of social deprivation on the course of lung disease such as chronic obstructive pulmonary disease and cystic fibrosis have suggested an association between low socioeconomic status and poorer prognosis $[1,2]$.

Idiopathic and heritable pulmonary arterial hypertension (IPAH and HPAH, respectively) are characterised by increasing pulmonary arterial pressure and, ultimately, right heart failure. Despite recent advances in treatment, IPAH and HPAH remain incurable diseases associated with high mortality and morbidity.

While the prognostic value of clinical variables has been well studied in IPAH, the influence of social factors on the course of disease is relatively unknown [3-5]. Recent studies from the USA and China suggest that social deprivation correlates with a more severe World Health Organization functional class at diagnosis in PAH as well as increased risk of death in patients with IPAH $[6,7]$.

This association has not been explored in a universal, free at point of care healthcare system such as that found in the UK. The aim of this study is to investigate the impact of social deprivation on prognosis in IPAH and HPAH patients throughout Scotland.

\section{Methods}

\section{Study design}

Ethical approval for this study was granted by the West Midlands - South Birmingham research ethics committee. Data were obtained on all patients with IPAH or HPAH diagnosed at the Scottish Pulmonary Vascular Unit (SPVU, Glasgow, UK) between January 24, 1992 and March 31, 2016. The SPVU is the only designated pulmonary hypertension centre in Scotland and has an exclusive remit for the investigation and management of pulmonary hypertension within the country. As a consequence, all cases with a confirmed diagnosis of IPAH and HPAH living in Scotland attend this unit, enabling as complete a capture as possible of cases from a well-defined geographical area.

Data were extracted from the local SPVU database and comprised both demographic and clinical variables recorded at point of diagnosis and outcome at point of censor. Postcodes for each subject were taken from both the date of diagnosis and the point of censor, defined as the date of death, last date of contact (if transferred to another unit or lost to follow up) or March 31, 2016. The demographic variables collected were age and sex. Clinical variables at diagnosis were body mass index (BMI), World Health Organization functional class (WHO-FC), right heart catheterisation values (mean pulmonary artery pressure, right atrial pressure (RAP), pulmonary arterial wedge pressure, mixed venous oxygen saturation $\left(S_{\mathrm{vO}_{2}}\right)$ and thermodilution cardiac output), 6-min walking distance (6MWD), N-terminal pro-brain natriuretic peptide (NT-proBNP), transfer factor of the lung for carbon monoxide (TLCO) and quality of life assessed using the Cambridge pulmonary hypertension outcome review (CAMPHOR) questionnaire [8]. Finally, treatment at point of censor was collected.

The Scottish Index of Multiple Deprivation (SIMD) is measured by the Scottish government and quantifies social deprivation based on data zones, a geographical unit comparable to a postcode [9]. Using pooled and weighted data from seven domains (employment, income, crime, housing, health, education and geographic access), each data zone is given a composite rank out of 6505 data zones. The composite rank was then converted to a quintile, with 1 assigned to the $20 \%$ most deprived data zones and 5 to the 20\% least deprived. SIMD data were published in 2004, 2006, 2009 and 2012 to reflect geographical changes in deprivation over time [9]. Postcode at point of diagnosis and censor for each subject were matched to the nearest iteration of SIMD data by date. For example, a subject diagnosed in 2009 and censored in 2014 would be assigned SIMD quintiles from 2009 for point of diagnosis and quintile from 2012 data for point of censor, respectively. The analysis was performed twice, using SIMD quintile from point of diagnosis and point of censor and the results compared.

In addition, social deprivation distribution data divided by sex, age and year were obtained from the National Records of Scotland [9]. Expected frequencies were calculated for each quintile of the dataset, matching by age and sex, to enable comparison of the social distribution of IPAH/HPAH patients compared to the Scottish population as a whole.

\section{Statistical analysis}

Statistical analysis was performed using R version 3.0.1 and Small STATA 11.1 (StataCorp, College Station, TX, USA). Potential associations between quintile and baseline variables were evaluated using multiple 
ANOVA for continuous outcome measures and Chi-squared tests of independence for dichotomous measures.

Cox regression was used to determine the association between survival and social deprivation quintile. In addition, baseline clinical variables were evaluated as predictors of survival using univariate Cox proportional hazard regression and the Kaplan-Meier method. Hazard ratios, 95\% confidence intervals and associated p-values were determined for each covariate. Multivariate analysis was performed to look for potential confounders with several models constructed to maximize data use where there were missing variables. Variables with few missing values (year of diagnosis, age, sex, BMI, RAP, cardiac output and $\mathrm{SvO}_{2}$ ) were included in all the models, whereas variables with more missing values (6MWD, TLCO, NT-proBNP and CAMPHOR score) were included only once in each model (see online supplementary material).

\section{Results}

Data were obtained on 280 subjects. Characteristics of these subjects at diagnosis are shown in table 1 . The cohort was $57 \%$ female, with an overall mean BMI of $28.7 \mathrm{~kg} \cdot \mathrm{m}^{-2}$. At diagnosis, mean age was 60 years and the majority $(83 \%)$ of patients were WHO-FC III or IV. Age, sex, BMI and CAMPHOR scores differed significantly across the quintiles. At point of censor there was no difference between the quintiles in the treatment received when assessed either as percentage on combination therapy or percentage on intravenous epoprostenol.

Between diagnosis and point of censor, 38 subjects changed deprivation quintile. This amounted to the cohort becoming 0.021 points less deprived at censor than at diagnosis. The analysis was performed twice using deprivation quintile at both point of diagnosis and point of censor, with no significant difference in results. Consequently, only analyses with deprivation quintile at point of censor are shown here. By the time of censor, 155 subjects had died, four had undergone transplantation and three were lost to

TABLE 1 Baseline characteristics for all subjects and across Scottish Index of Multiple Deprivation quintiles

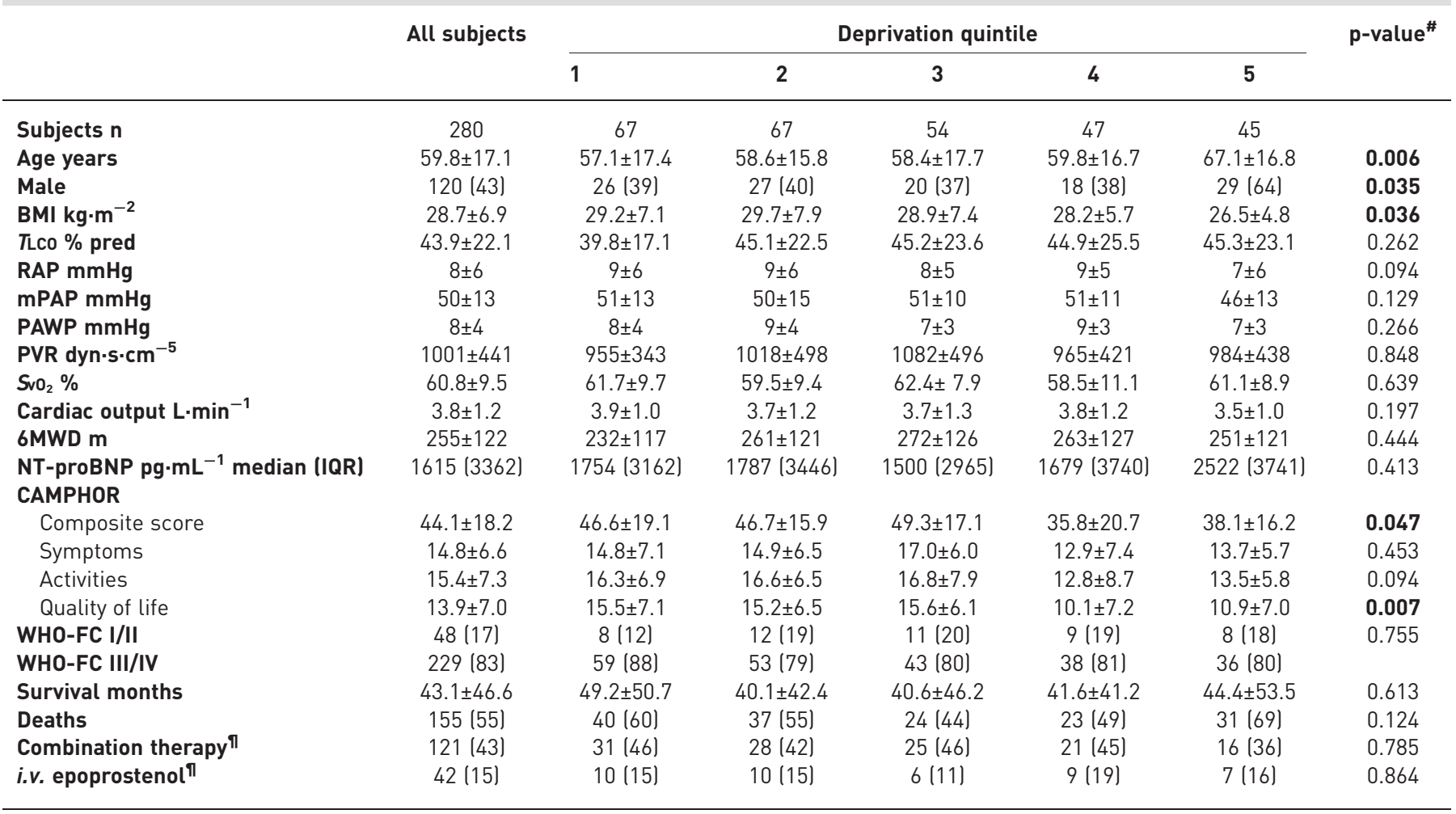

Data are presented as mean \pm SD or $n(\%)$, unless otherwise stated. Quintile 1 represents the greatest deprivation and quintile 5 represents the least deprived subjects. BMI: body mass index; TLCO: transfer factor of the lung for carbon monoxide; RAP: right atrial pressure; mPAP: mean pulmonary artery pressure; PAWP: pulmonary arterial wedge pressure; PVR: pulmonary vascular resistance; Svo : mixed venous oxygen saturation; 6MWD: 6-min walking distance; NT-proBNP: N-terminal pro-brain natriuretic peptide; IQR: interquartile range; CAMPHOR: Cambridge Pulmonary Hypertension Outcome Review; WHO-FC: World Health Organization functional class. ${ }^{\#}$ : p-value for trend over quintiles; ף: treatment at point of censor. Bold type represents statistical significance. 
follow-up. Kaplan-Meier analysis of the cohort showed 1-year, 3-year and 5-year survival as $82 \%$, $53 \%$ and $41 \%$, respectively, with no difference seen between deprivation quintiles.

Cox regression analysis was used to examine the relationship between social deprivation and survival, and the results are shown in table 2. No significant association was found despite adjustment for time, age and sex. In contrast, the same univariate analysis using baseline demographic and clinical variables did show prognostic significance for several of the variables analysed, as detailed in table 3. Several multivariate models were constructed to look for potential confounders using time of enrolment, age, sex, BMI and the significant variables from table 3 . None produced a significant relationship between deprivation and survival. The results of this analysis are included in the online supplementary material.

Since sex varies significantly across the deprivation quintiles, the analysis on baseline characteristics and predictors of mortality was repeated on males and females separately. This approach also failed to show a convincing association between social deprivation and survival, and the results of this analysis are shown in the online supplementary material.

Finally, figure 1 shows the observed and expected social deprivation distributions of the IPAH/HPAH dataset. The expected distribution of social deprivation for the dataset is derived from the distribution of the Scottish population as a whole, matched by age and sex. The observed distribution was more heavily skewed toward the more deprived quintiles than would be expected based on the matched age-sex distribution of the Scottish population (Chi-squared 16.16, $\mathrm{df}=4, \mathrm{p}=0.003$ ).

\section{Discussion}

In this retrospective cohort study, there was no association between social deprivation measured by the SIMD and either WHO-FC or survival among Scottish patients with IPAH and HPAH. The addition of prognostic demographic and clinical variables to multivariate models did not alter the findings. Similarly, analyses using social deprivation quintile assessed at both point of diagnosis and point of censor produced nonsignificant results. However, the data showed that survival was dependent on other well-recognised prognostic variables such as RAP, 6MWD, NT-proBNP and TLCO [3-5]. There was variation in age, BMI and sex with social deprivation, but no difference in intensity of treatment received. In addition, there was an over-representation of HPAH and IPAH subjects among the more socially deprived quintiles when compared with the age- and sex-matched distribution for the Scottish population as a whole.

This study is the first to examine the relationship between socioeconomic status, IPAH/HPAH severity and prognosis in a national cohort of patients and in a healthcare system free at the point of access. Our subject population captures this information for the entire Scottish population, while holding constant healthcare provision (the National Health Service (NHS)) and the method of diagnosis (the SPVU). Additionally, this dataset permits an evaluation of the impact of social deprivation on equity of referral for patients with IPAH in Scotland.

This study benefited from a robust metric for social deprivation, the SIMD. The SIMD encompasses several aspects of social deprivation, thereby providing a more complete picture of social wellbeing beyond income or education measures as used in other studies. Although the study was limited by a lack of subject-level socioeconomic data, the SIMD is based on a small geographic unit (postcode) and is therefore relatively specific to each patient.

The primary limitation of this study is its retrospective nature. Therefore, the ability of the study to assess time-related associations or causations between social deprivation and survival is limited. Furthermore, its conclusions rely on minimal change in diagnostic practice over time. The latter is unlikely to be a significant problem as the unit has had the same medical director over this period and the diagnostic

TABLE 2 Hazard ratios of all-cause mortality across Scottish Index of Multiple Deprivation quintiles

\begin{tabular}{|c|c|c|c|c|c|c|}
\hline & \multicolumn{5}{|c|}{ Deprivation quintile } & $\mathrm{p}$-value for trend \\
\hline $\begin{array}{l}\text { Unadjusted } \\
\text { p-value }\end{array}$ & $\begin{array}{c}0.83(0.52-1.33) \\
0.44\end{array}$ & $\begin{array}{c}0.88(0.54-1.43) \\
0.61\end{array}$ & $\begin{array}{c}0.74(0.34-1.28) \\
0.28\end{array}$ & $\begin{array}{c}0.75(0.44-1.30) \\
0.31\end{array}$ & 1.0 (ref.) & 0.81 \\
\hline $\begin{array}{l}\text { Adjusted for time, age and sex } \\
p \text {-value }\end{array}$ & $\begin{array}{c}1.37(0.84-2.24) \\
0.21\end{array}$ & $\begin{array}{c}1.15(0.70-1.88) \\
0.58\end{array}$ & $\begin{array}{c}1.39(0.79-2.45) \\
0.26\end{array}$ & $\begin{array}{c}1.05(0.60-1.85) \\
0.86\end{array}$ & 1.0 (ref.) & 0.23 \\
\hline
\end{tabular}

Data are presented as hazard ratio $(95 \%$ CI), unless otherwise stated. Quintile 1 represents the most deprived subjects and quintile 5 represents the least deprived. $n=280$. 


\begin{tabular}{|c|c|c|c|}
\hline & Subjects $\mathrm{n}$ & Hazard ratio $(95 \% \mathrm{CI})$ & p-value \\
\hline Age years & 280 & $1.04(1.03-1.05)$ & $<0.001$ \\
\hline Male & 280 & $1.59(1.16-2.18)$ & 0.004 \\
\hline $\mathrm{BMI} \mathrm{kg} \cdot \mathrm{m}^{-2}$ & 263 & $0.99(0.96-1.02)$ & 0.4 \\
\hline TLco $\%$ pred & 237 & $0.97(0.96-0.98)$ & $<0.001$ \\
\hline RAP mmHg & 275 & $1.03(1.0-1.06)$ & 0.04 \\
\hline mPAP $\mathrm{mmHg}$ & 276 & $0.99(0.98-1.01)$ & 0.4 \\
\hline PAWP $\mathrm{mmHg}$ & 262 & $0.99(0.95-1.04)$ & 0.8 \\
\hline Cardiac output L.min ${ }^{-1}$ & 267 & $0.84(0.72-0.98)$ & 0.03 \\
\hline $\mathrm{SvO}_{2} \%$ & 235 & $0.98(0.96-0.99)$ & 0.05 \\
\hline 6MWD m & 202 & $0.995(0.994-0.996)$ & $<0.001$ \\
\hline $\begin{array}{l}\log _{10} \text { NT-proBNP } \\
\text { CAMPHOR }\end{array}$ & 183 & $2.38(1.60-3.54)$ & $<0.001$ \\
\hline Composite score & 98 & $1.02(1.00-1.04)$ & 0.04 \\
\hline Symptoms & 98 & $1.02(0.98-1.07)$ & 0.3 \\
\hline Activities & 98 & $1.08(1.03-1.12)$ & $<0.001$ \\
\hline Quality of life & 98 & $1.02(0.98-1.06)$ & 0.3 \\
\hline
\end{tabular}

BMI: body mass index; TLCO: transfer factor of the lung for carbon monoxide; RAP: right atrial pressure; mPAP: mean pulmonary artery pressure; PAWP: pulmonary arterial wedge pressure; $\mathrm{SvO}_{2}$ : mixed venous oxygen saturation; 6MWD: 6-min walking distance; NT-proBNP: N-terminal pro-brain natriuretic peptide; CAMPHOR: Cambridge Pulmonary Hypertension Outcome Review. Bold type represents statistical significance.

definition of IPAH has been constant. A further issue is that the clinical use of prognostic indicators such as CAMPHOR score and NT-proBNP changed over the course of the study, resulting in variation of measured subject data across time. Therefore, the multivariate analyses using these indicators were limited, as inclusion of all the available variables would impair the analysis by excluding too many subjects due to missing data The analysis presented in this study does not exclude the possibility that an impact of social deprivation on prognosis has not been detected because of the limited size of the dataset. Conversely, creation of a larger cohort would add further potential bias because of expansion in geographical location, centre practice or time.

These results differ from those reported by TALWAR et al. [6] and Wu et al. [7], studies from the USA and China, respectively. TALwar et al. showed an association between lower household income and worse WHO-FC at presentation and concluded that healthcare costs in their setting probably lead to a delay in referral. Wu et al. showed an association between lower socioeconomic status (derived from educational level, income, occupation and medical reimbursement rate) and poorer survival despite adjusting for age, sex and disease severity. The study population presented by Wu et al., with a mean age of 36 years is very different from that seen in Scotland and study populations described in other registries from Western countries [10]. This suggests that many older sufferers of pulmonary hypertension in China are not being

FIGURE 1 Observed and expected social deprivation distributions of the idiopathic pulmonary arterial hypertension/hereditary pulmonary arterial hypertension dataset. Quintile 1 represents the greatest social deprivation.

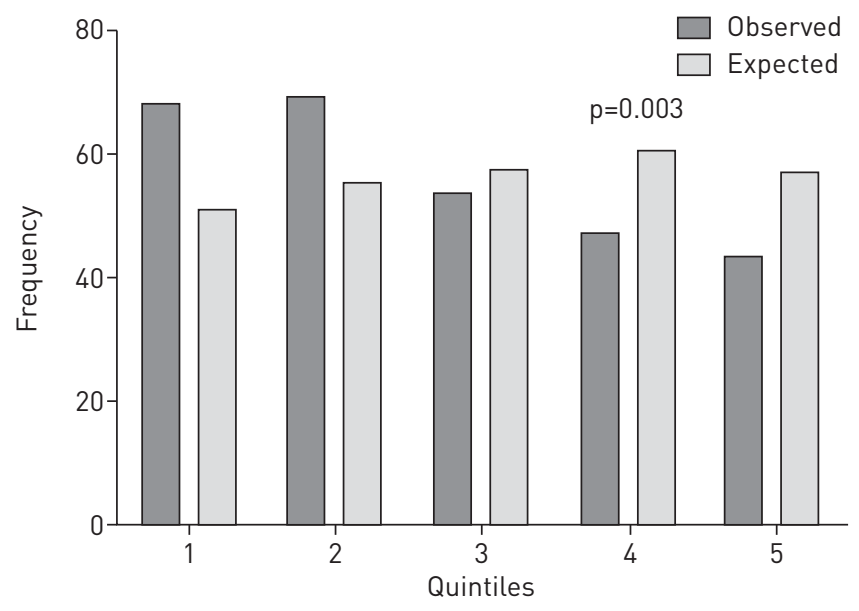


referred and that the generalisability of their conclusions to Western practice is limited. Wu et al. [7] did not see a relationship between WHO-FC and socioeconomic status at diagnosis, but unlike this study did show that subjects with lower socioeconomic status received less treatment for pulmonary hypertension, suggesting that healthcare costs were having an impact on prognosis.

A key difference between the current study and previously published data $[6,7]$ is in healthcare infrastructure. In Scotland, access to healthcare and subsequent treatment are paid for by national taxation and are free at the point of delivery as part of the NHS. This removes much of the financial barrier between low socioeconomic status and access to medical services. The lack of the association between socioeconomic status and either WHO-FC or survival in our population attests to the efficacy of this approach and supports the conclusions of both TALWAR et al. [6] and WU et al. [7]. In addition, it suggests that it is not low socioeconomic status per se that worsens prognosis in IPAH/HPAH, but rather access to appropriate care. A second difference, which complicates interpretation of these contrasting study outcomes is that treatment for pulmonary hypertension in Scotland is only available from one centre, the SPVU, to which all patients in the country with potential pulmonary hypertension are referred. This centralisation of care enhances the likelihood of consistency of care regardless of the patient's postcode. However, this approach does not overcome social barriers both to seeking healthcare in the first instance and to subsequent appropriate referral to the pulmonary hypertension centre, and can only partially explain the lack of prognostic impact of social deprivation seen in this study.

Previous studies examining the effect of social deprivation on access to care and survival for other diseases within Scotland have shown heterogeneous outcomes. Several studies have found that SIMD score is not related to disease severity at presentation or subsequent prognosis. These include renal transplantation [11], the management of small renal masses [12] and myelodysplasia receiving supportive treatment [13]. Conversely, prognosis in other conditions is linked to social deprivation, such as mortality in chronic kidney disease [14], diabetes mellitus [15] and primary glomerulonephritis [16]. The reasons for this disparity require further study. It may be related in part to the prevalence of a condition and variations in the resources available to diagnose and treat within a region. It may also be related to the ability of the patient to engage with some forms of treatment because of factors such as comorbidity, which is more common with increased deprivation. An interesting example which in some aspects supports these hypotheses is that of colorectal cancer, where deprivation is not associated with treatment delay or more advanced disease stage at presentation, but is associated ultimately with poorer survival [17]. These data suggest that the findings within this study in pulmonary hypertension are possibly a consequence of the universal coverage of the NHS, the centralisation of pulmonary hypertension care within Scotland and the demographics of the disease itself.

The lack of a socioeconomic barrier to referral in Scotland is also suggested by the preponderance of patients in this population who fall into the more deprived quintiles. The reason for this distribution is not obvious. It is possible that environmental factors in more socially deprived households unidentified in this study are leading to a higher incidence of IPAH. Alternatively, the presence of chronic disease over several years prediagnosis may have an impact on employment prospects and cause lower socioeconomic status. Third, more deprived patients may have a higher burden of comorbidities and either present earlier because of reduced functional reserve, augmenting symptoms, or more frequent contact with medical services. This theory is supported by the finding that subjects in more deprived quintiles tended to be younger with a higher BMI than their more affluent peers. A further possibility is a referral bias to specialised care if patients live near city centres, which tend to be areas of greater social deprivation in Scotland, but no geographical variation in rates of IPAH is seen across the Scottish health boards [9].

The variation of quality of life and BMI with social deprivation is predictable and unsurprising [18, 19]. The variation of social deprivation with age and sex are largely driven by a preponderance of older males in the least socially deprived quintile. Some, but not all of this could be due to worse survival of more socially deprived males due to comorbidities such as ischaemic heart disease.

\section{Conclusion}

This study demonstrates that social deprivation is not associated with disease severity or survival in Scottish patients with IPAH and HPAH. Furthermore, the patient population is more deprived than the Scottish population as a whole. These findings suggest that low socioeconomic status is neither a prognostic factor nor a barrier to treatment for IPAH and HPAH patients under the system practised in the UK NHS in Scotland.

\section{References}

1 Eisner MD, Blanc PD, Omachi TA, et al. Socioeconomic status, race and COPD health outcomes. J Epidemiol Community Health 2011; 65: 26-34. 
2 O'Connor GT, Quinton HB, Kneeland T, et al. Median household income and mortality rate in cystic fibrosis Pediatrics 2003; 111: e333-e339.

3 Lee W-TN, Ling Y, Sheares KK, et al. Predicting survival in pulmonary arterial hypertension in the UK. Eur Respir J 2012; 40: 604-611.

4 Benza RL, Miller DP, Gomberg-Maitland M, et al. Predicting survival in pulmonary arterial hypertension: insights from the Registry to Evaluate Early and Long-Term Pulmonary Arterial Hypertension Disease Management (REVEAL). Circulation 2010; 122: 164-172.

5 Howard LS. Prognostic factors in pulmonary arterial hypertension: assessing the course of the disease. Eur Respir Rev 2011; 20: 236-242

6 Talwar A, Sahni S, Talwar A, et al. Socioeconomic status affects pulmonary hypertension disease severity at time of first evaluation. Pulm Circ 2016; 6: 191-195.

7 Wu W-H, Yang L, Peng F-H, et al. Lower socioeconomic status is associated with worse outcomes in pulmonary arterial hypertension. Am J Respir Crit Care Med 2013; 187: 303-310.

8 McKenna SP, Doughty N, Meads DM, et al. The Cambridge Pulmonary Hypertension Outcome Review (CAMPHOR): a measure of health-related quality of life and quality of life for patients with pulmonary hypertension. Qual Life Res 2006; 15: 103-115.

9 Scottish Executive. Scottish Index of Multiple Deprivation. Scottish Index Mult Deprivation 2012 Summ Tech Rep 2013.

10 Hoeper MM, Gibbs JS. The changing landscape of pulmonary arterial hypertension and implications for patient care. Eur Respir Rev 2014; 23: 450-457.

11 Aitken E, Dempster N, Ceresa C, et al. The impact of socioeconomic deprivation on outcomes following renal transplantation in the West of Scotland. Transplant Proc 2013; 45: 2176-2183.

12 Leonard M, Tait CD, Gillan AS, et al. Impact of multiple deprivations on detection, progression and interventions in small renal masses (less than $4 \mathrm{~cm}$ ) in a population based study. Eur J Surg Oncol 2013; 39: 1157-1163.

13 Mastaglio F, Bedair K, Papaemmanuil E, et al. Impact of socioeconomic status on disease phenotype, genomic landscape and outcomes in myelodysplastic syndromes. Br J Haematol 2016; 174: 227-234.

14 Solbu MD, Thomson PC, Macpherson S, et al. Serum phosphate and social deprivation independently predict all-cause mortality in chronic kidney disease. BMC Nephrol 2015; 16: 194.

15 Walker J, Halbesma N, Lone N, et al. Socioeconomic status, comorbidity and mortality in patients with type 2 diabetes mellitus in Scotland 2004-2011: a cohort study. J Epidemiol Community Health 2016; 70: 596-601.

16 McQuarrie EP, Mackinnon B, Bell S, et al. Multiple socioeconomic deprivation and impact on survival in patients with primary glomerulonephritis. Clin Kidney J 2017; 10: 49-54.

17 Paterson HM, Mander BJ, Muir P, et al. Deprivation and access to treatment for colorectal cancer in southeast Scotland 2003-2009. Colorectal Dis 2014; 16: O51-O57.

18 Tyrrell J, Jones SE, Beaumont R, et al. Height, body mass index, and socioeconomic status: mendelian randomisation study in UK Biobank. BMJ 2016; 352: i582.

19 Mielck A, Vogelmann M, Leidl R. Health-related quality of life and socioeconomic status: inequalities among adults with a chronic disease. Health Qual Life Outcomes 2014; 12: 58. 